\title{
Cathodoluminescence Mapping of Chondrules and their Constituents: Identification of Zoning Patterns in Olivine and Chondrules and Implications for their Formation History and Parent Bodies Processes.
}

Juliane Gross ${ }^{1,2,3}$, and Tasha L. Dunn ${ }^{4}$.

1. Department of Earth and Planetary Sciences, Rutgers University, Piscataway NJ 08854.

2. Department of Earth and Planetary Sciences, The American Museum of Natural History, New York NY 10024.

3. Lunar and Planetary Institute, Houston TX 77058.

4. Department of Geology, Colby College, Waterville ME 04901

The formation of chondrules and their constituents is highly debated - whether formed from a vapor or a liquid. Two general models have been proposed: 1) chondrule formation in energetic events occurring on or near planetesimals; or 2) chondrule formation occurring independent of planetesimals through nebular processes (e.g. [1-5]). These theories can be investigated by examining the individual minerals, such as olivine, within chondrules that respond texturally and compositionally to changes in their formation environments. Therefore, a record of their formation history and formation processes is preserved in the olivine crystal growth stratigraphy [6] and can be accessed by examination and elemental X-ray mapping of the olivine zoning patterns for some elements [e.g., 7-9]. However, major elements such as $\mathrm{Fe}, \mathrm{Mg}$, and Si diffuse rapidly within the olivine structure eliminating fine scaled zoning patterns and thus making it impossible to access the information about formation history through elemental x-ray mapping [10].

Cathodoluminescence (CL), however, can reveal subtle changes in zoning structures and textural varieties within olivine that cannot be observed with any other mapping technique [7]. In the past this technique has been used to reveal oscillatory zoning in olivine grains within the matrix of carbonaceous chondrites [7, 8] which suggested formation from a melt [8]. In this work we have begun a systematic CL study of the carbonaceaous chondrite groups CV3 and CK3 as well as unequilibrated ordinary chondrites LL3. It is debated whether the CV and CK chondrites come from a single parent body or represent unique parent bodies [11,12]. A systematic CL study of olivine grains within chondrules of the $\mathrm{CV}$ and CK chondrites could help to place constraints on the formation history of the parent body(s) from which these chondrites originated. In addition, the detailed examination of olivine crystal stratigraphy across different meteorite groups and different chondrule types will show if similar features are present, and thus allow us to place constraints on the formation processes and history of chondrules.

For this study we used the Cathodoluminescence Microanalyser xCLent that is incorporated into the JEOL-8200 Electron Microprobe (EPMA) at Rutgers University. Chondrules were mapped with 15kV accelerating voltage, 20nA beam current, focused beam, and a dwell time of 30ms. X-ray element maps were obtained at the same time to link potential zoning patterns of elements with the observed CL patterns. RGB (RedGreenBlue) maps of the CL spectra were created to reveal 1) complicated zoning patterns within individual olivine crystals (Fig. 1a), and 2) oscillatory zoning patterns of the chondrules themselves (Fig. 1b). Olivine within the core of the chondrule show a red CL spectra as well as olivine towards the rim. Olivine located in the middle of the chondrule show a blueish to purple CL color. Each color zone corresponds to eithers a slightly different chemistry and/or defects of the crystal structure. More work has to be done to quantify these difference in the CL color. Cathodoluminscence is an easy 
way to prospect for zoned chondrules and zoned olivine within meteorites that do not show evidence of zonation based solely on elemental X-ray and BSE images.

\section{References:}

[1] F.J. Ciesla, Chondrites and the Protoplanetary Disk (2005), Vol. 341, p.811-820’

[2] R.H. Hewins, H.C. Connolly, Jr., G.E. Lofgren and G. Libourel, Chondrites and the Protoplanetary Disk (2005), Vol. 341, p.286-316.

[3] R.H. Jones, J.N. Grossman, and A.E. Rubin, Chondrites and the Protoplanetary Disk (2005), Vol. 341, p.251-285.

[4] E.R.D. Scott and N. Krot, Chondrites and the Protoplanetary Disk (2005), Vol. 341, p.15-53.

[5] C.M. O’D. Alexander, J.N. Grossman, D.S. Ebel, F.J. Ciesla, Science (2008), Vol. 320, p.1617-1619.

[6] M. Streck, Reviews in Mineralogy \& Geochemistry, Vol. 69 (2008), p.595-622.

[7] I.M. Steele, J.V. Smith, and C. Skirius, Nature (1985), Vol. 313, p.294-297.

[8] I.M. Steele, American Mineralogist (1995). Vol. 80, p.823-832.

[9] M. Miyamoto, D.S. McKay, G.A. McKay, and M.B. Duke, Journal of Geophysical Research (1986), Vol. 91, p.12804-12816.

[10] F. Costa, R. Dohmen, and S. Chakraborty, Reviews in Mineralogy \& Geochimistry, Vol. 69, p.545594.

[11] M.K. Weisberg, T.J. McCoy, and A.N. Krot (2006), in “Meteoritics and the Early Solar System”, $2^{\text {nd }}$ ed. Tucson: University of Arizona Press. p.19-52

[12] R.C. Greenwood, I.A. Franchi, A.T. Kearsley, O. Alard, Geochimica et Cosmochimica Acta (2010) Vol. 74, p.1684-1705.
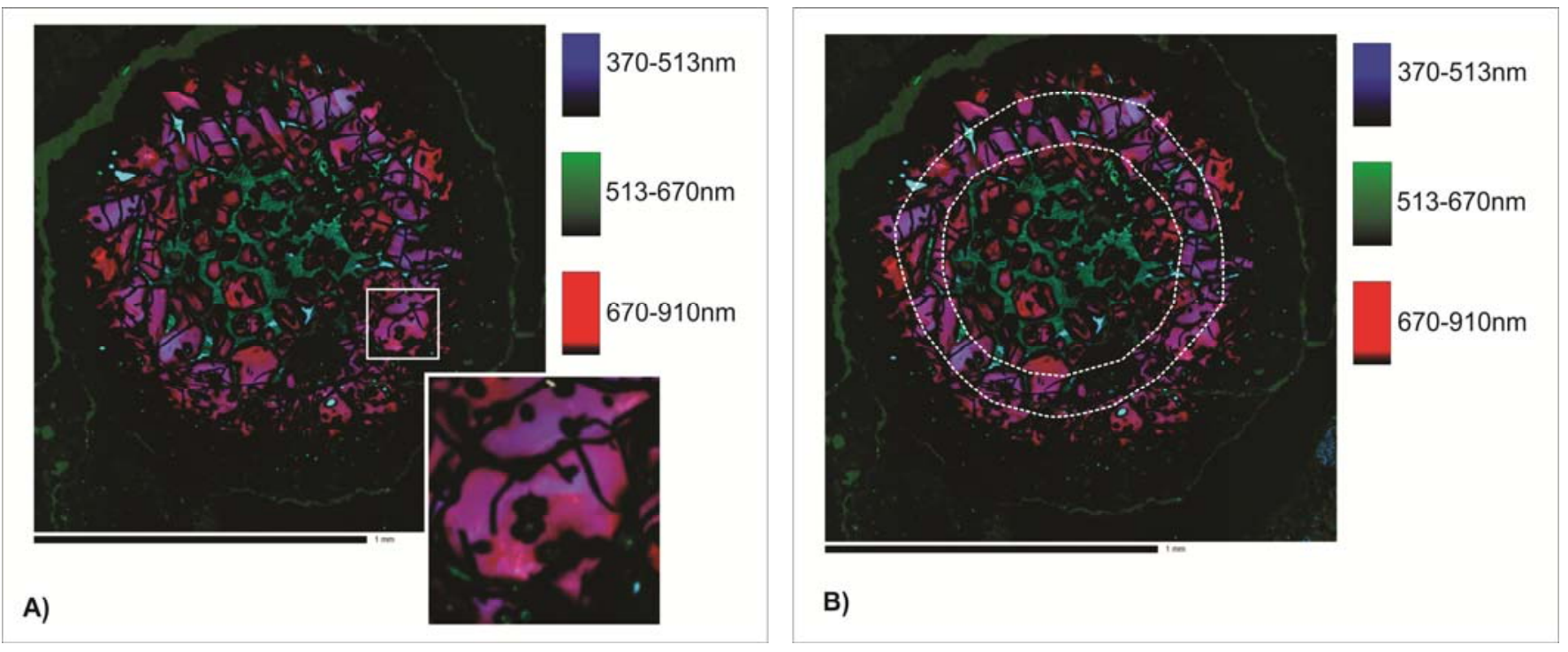

Figure 1a. RedGreenBlue map of CL wavelengths (Red=670-910nm; Green=513-670nm; Blue=370$513 \mathrm{~nm})$. Zoning structures of individual olivine grains within the chondrule can be observed.

Figure 1b. RedGreenBlue map of CL wavelengths (Red=670-910nm; Green=513-670nm; Blue=370$513 \mathrm{~nm})$. Oscillatory zoning of the chondrule itself is observed. Within the core of the chondrule olivine shows red color, followed by a zone of grains showing purple CL color, outlined in the white dotted lines, which is followed by a zone of olivine that show red CL color. The outermost rim is too FeO rich to display CL colors. The matrix in both figures is green. 